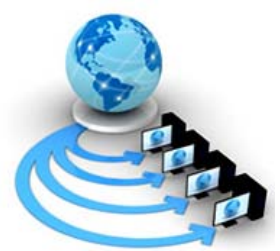

Volume 9, No. 1, January-February 2018

International Journal of Advanced Research in Computer Science

RESEARCH PAPER

\title{
TYPE-2 FUZZY MULTIPLICATION WAVELET NEURAL NETWORK MODEL DESIGN FOR CLASSIFICATION OF BREAST CANCER
}

\author{
Sevcan Yilmaz Gunduz \\ Department of Computer Engineering \\ Anadolu University \\ Eskisehir, Turkey
}

\begin{abstract}
Breast cancer is one of the most common types of cancer. For this reason, it is very important to diagnose breast cancer. In this paper, a type-2 fuzzy multiplication wavelet neural network model is proposed to classify the Wisconsin Breast Cancer dataset. In this model, Shannon wavelet function is used as the type-2 membership function and the multiplication of the Shannon wavelet functions is used in the conclusion part of the rules. The results of proposed model is compared with type-1 fuzzy multiplication wavelet neural network, multilayer perceptron network, radial basis function network, Bayesian network learning, and decision tree algorithm. It can be seen that proposed type-2 fuzzy multiplication wavelet neural network model gives the best results among these algorithms.
\end{abstract}

Keywords: type-2 fuzzy system; fuzzy wavelet neural network; wavelet; classification; breast cancer

\section{INTRODUCTION}

Breast cancer is one of the leading cancer types that cause women to die. Even a medical specialist is sometimes difficult to diagnose the cancer after performing various tests. For this reason, diagnosing breast cancer automatically has an important place among medical problems. In the literature, various studies have been done towards this aim [1-8]. In [1], support vector machines and radial basis function networks are used for breast mass classification based on cytological patterns. In [2], association rules are used for dimension reduction for breast cancer dataset and the data obtained is used for input to neural network in order to breast cancer classification of breast cancer. One of the most recent papers about the computer aided breast cancer diagnosis system is proposed in [3]. In this paper, some statistical feature selection methods are applied to breast cancer dataset in order to select categorical features and these features are refined by particle swarm optimization and bagged decision tree is used for classification. In [4], a feed forward artificial neural network is used for medical diagnosis and particle swarm optimization is used for training this network. In [5], interval type-2 fuzzy logic system and wavelet transform are used for classification of medical data. In [6], s an integration of fuzzy standard additive model with genetic algorithm is proposed to classify the healthcare data. In [7], a new method is proposed in order to selecting genes to mediating some certain cancer disease. In [8], a diagnostic system using neural networks is proposed for classification of clinical datasets and differential evolution and particle swarm optimization is used for training of the model.

Fuzzy neural networks call the combination of neural networks and fuzzy systems [9-12] and they are used many application areas. In the design of fuzzy neural networks, a training algorithm is used to construct fuzzy rules and membership functions. However, sometimes the measured data of the system can be have some uncertainties and these uncertainties are important in the fuzzy system design and must be considered. Uncertainty in type-1 fuzzy neural networks is achieved through membership functions. However, sometimes the measurements can have some uncertainties caused by devices or noise in the environment. Type-2 fuzzy neural networks are used to overcome such uncertainties [13, 14]. Type-2 fuzzy systems can reduce the effects of uncertainties [15]. Type-2 fuzzy systems increase the computational complexity according to type-1 fuzzy logic systems. However, type-2 fuzzy logic systems give better results in many areas. They have been applied to many different applications such as identification of nonlinear systems [1621], control [22, 23], time series prediction [24], system modeling [20, 25, 26], stock price prediction [27] and control of mobile robots [28, 29]. In [30], a review of type-2 fuzzy logic applications is presented for pattern recognition. In this paper, type-2 wavelet membership functions were used in fuzzy rules.

Another model for prediction and classification of various systems is fuzzy wavelet neural networks (FWNN), which combines fuzzy neural networks with wavelet functions. Wavelet functions increases the performance of the fuzzy neural networks [31-33]. Fuzzy wavelet neural networks have been used in various applications. This paper proposes a type-2 fuzzy multiplication wavelet neural network model to classification of breast cancer. The model gets its idea from [32] and [31] in which three new FWNN models are presented for prediction and identification of nonlinear dynamical systems. The new model is named as type-2 fuzzy multiplication wavelet neural network (T2FWNN) and used for identification of dynamical plants in [34]. In this paper, the model in [34] is updated to type-2 fuzzy multiplication wavelet neural network (T2FMWNN) in order to achieve classification problems and is used for classification of breast cancer dataset. In the proposed T2FMWNN model, the antecedent with type-2 Shannon wavelet functions. The consequent rule output functions are developed using multiplication of dilated and translated versions of Shannon wavelet functions of the input variables as in FWNN-M model introduced in [32]. However in [32] and [31], Mexican hat wavelet function is used in type-1 FWNN models for system identification. In addition, [35], a type-2 wavelet fuzzy neural network model using Mexican 
hat wavelet functions in used in the conclusion part of the rules is used to time series prediction. The proposed new T2FMWNN model gives better results according to type-1 FMWNN (T1FMWNN), multilayer perceptron (MLP) network, radial basis function network (RBF) and FURIA in breast cancer classification.

\section{TYPE-2 FUZZY MULTIPLICATION WAVELET NEURAL NETWORK MODEL}

Firstly, the components of type-2 fuzzy system will be explained. Type-2 fuzzy systems are characterized by fuzzy IF-THEN rules, the fuzzy sets in the antecedent and the consequent parts of the rules are type-2. A typical type-2 fuzzy system is shown in Fig. 1. Similar to a type-1 fuzzy system, a type-2 fuzzy system includes a fuzzifier unit, a fuzzy rule base, a fuzzy inference engine, type-reducer and defuzzifier units.

This paper proposes type-2 fuzzy multiplication wavelet neural network (T2FMWNN) model for classification of breast cancer dataset. T2FMWNN embeds the useful properties of type-2 fuzzy system with time and frequency localization properties of wavelets into the framework of an adaptive neural network so that training methods of neural network technologies can be easily applied. T2FMWNN model structure has type-2 fuzzy sets as membership functions and multiplication of wavelet functions in the consequent part of the rules. Fig. 2 show the structure of the T2FMWNN model.

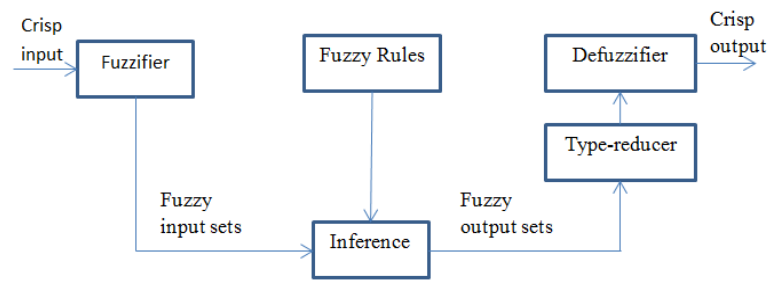

Fig. 1. Type-2 fuzzy logic system [34]

The T2FMWNN model has totally six major layers. The fuzzy rules in this fuzzy system are in the following form: IF $x_{1}$ is $\Psi_{1 i}$ and $x_{2}$ is $\Psi_{2 i}$ and $\ldots$ and $x_{n}$ is $\Psi_{n i}$

$$
\text { THEN } g_{l}(x)
$$

where $x_{1}, x_{2}, \ldots, x_{n}$ are input variables that represents the states of the plant to be identified, $\Psi_{i j}$ is the $j$ th wavelet type membership function (MF) for the ith input and $g_{l}(x)$, which is a function of inputs, is the lth output of fuzzy rule.

In this paper, translated and dilated version of Shannon wavelet function is used as membership function and multiplication of the wavelet functions are used in conclusion part of the rules.

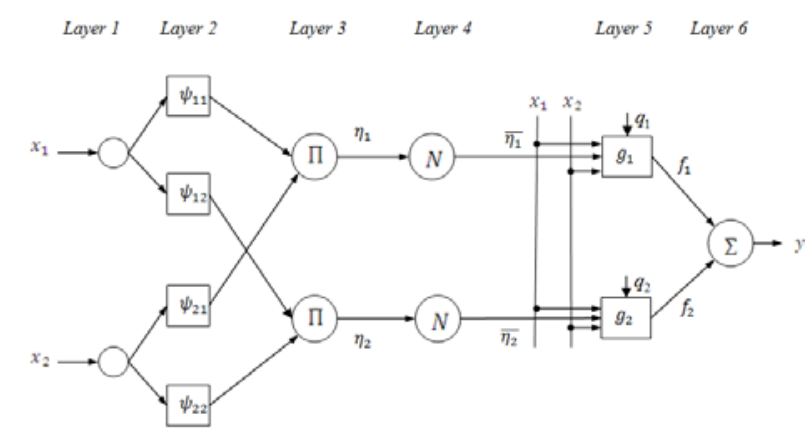

Fig. 2. Structure of the T2FMWNN model [34]

For simplicity and understandability, we will explain the models for two inputs and two membership functions for these inputs.

The first layer is input layer. It transmits inputs, $x_{1}, x_{2}$ to second layer. Second layer is fuzzification layer. Interval type-2 wavelet membership functions are used in this layer. Lower and upper membership functions are defined as follows:

$$
\begin{aligned}
& \Psi_{i j}=\left(\underline{\Psi}_{i j}, \bar{\Psi}_{i j}\right) \\
& \underline{\Psi}_{i j}=\frac{\sin \left(\frac{x_{j}-\underline{\mu}_{i j}}{\underline{\sigma}_{i j}}\right)}{\left(\frac{x_{j}-\underline{\mu}_{i j}}{\underline{\sigma}_{i j}}\right)} \\
& \bar{\Psi}_{i j}=\frac{\sin \left(\frac{x_{j}-\bar{\mu}_{i j}}{\bar{\sigma}_{i j}}\right)}{\left(\frac{x_{j}-\bar{\mu}_{i j}}{\bar{\sigma}_{i j}}\right)}
\end{aligned}
$$

where $i=1,2$ and $j=1,2$

Both center, $\underline{\mu}_{i j} \quad \bar{\mu}_{i j} \quad$ and standard deviation, $\underline{\sigma}_{i j} \bar{\sigma}_{i j}$ parameter can take proper initial values and these values are updated during the training phase.

Third layer is fuzzy rule layer. Multiplication operator is used in this layer.

$$
\begin{aligned}
& \underline{\eta}_{j}=\underline{\Psi}_{1 j}\left(x_{1}\right) \underline{\Psi}_{2 j}\left(x_{2}\right) \\
& \bar{\eta}_{j}=\bar{\Psi}_{1 j}\left(x_{1}\right) \bar{\Psi}_{2 j}\left(x_{2}\right)
\end{aligned}
$$

In the fourth, fifth and sixth layers, the overall output value is calculated as summation of weighted output values of each rule. In the following formulas, $q_{j}$ is design factors for lower and upper membership functions:

$$
\begin{aligned}
& y=\frac{\sum_{j=1}^{2} q_{j} \underline{\eta}_{j} g_{j}}{\sum_{j=1}^{2} \underline{\eta}_{j}}+\frac{\sum_{j=1}^{2}\left(1-q_{j}\right) \bar{\eta}_{j} g_{j}}{\sum_{j=1}^{2} \bar{\eta}_{j}} \\
& g_{j}=w_{j} \prod_{i=1}^{n} \frac{\sin \left(\frac{x_{i}-b_{i j}}{c_{i j}}\right)}{\left(\frac{x_{i}-b_{i j}}{c_{i j}}\right)}+p_{j}
\end{aligned}
$$

Conventionally, design factors are determined for once for the whole rule base in the previous papers $[16,18,25$, 
36]. But in this study, for each rule, different design factors are used and this adjusts the lower and upper parts. In the simulations part, the results of T2FMWNN model and also the results of T1FMWNN model are compared.

To obtain the optimal T2FMWNN model parameters for classification problems, a gradient based algorithm is chosen. In these algorithms, firstly a cost function is chosen to be minimized, so that T2FMWNN model finds the optimal parameters. Here, the mean square error (MSE) is selected as objective function:

$$
E=\frac{1}{N} \sum_{k=1}^{N}\left(y-y_{d}\right)^{2}
$$

where $\mathrm{N}$ is the number of input-output pairs of the function to be approximated, $\mathrm{y}_{\mathrm{d}}$ is the desired output, and $\mathrm{y}$ is the T2FMWNN model output.

In this study, a gradient based algorithm DavidonFletcher-Powel (DFP) [37] method is used to train the model. Gradients of the MSE with respect to membership function parameters of the T2FMWNN models are calculated by the following formulas:

$$
\begin{aligned}
& \frac{\partial E}{\partial \mu_{i j}}=\frac{\partial E}{\partial y} \frac{\partial y}{\partial \Psi_{i j}}\left(\frac{-\cos \left(\left(x_{j}-\mu_{i j}\right) / \sigma_{i j}\right)}{x_{j}-\mu_{i j}}+\frac{q \sin \left(\left(x_{j}-\mu_{i j}\right) / \sigma_{i j}\right)}{\left(x_{j}-\mu_{i j}\right)^{2}}\right) \\
& \frac{\partial E}{\partial \sigma_{i j}}=\frac{\partial E}{\partial y} \frac{\partial y}{\partial \Psi_{i j}}\left(\frac{-\cos \left(\left(x_{j}-\mu_{i j}\right) / \sigma_{i j}\right)}{\sigma_{i j}}+\frac{\sin \left(\left(x_{j}-\mu_{i j}\right) / \sigma_{i j}\right)}{x_{j}-\mu_{i j}}\right)
\end{aligned}
$$

$\frac{\partial E}{\partial y}=\frac{2}{N} \sum_{k=1}^{N}\left(y-y_{d}\right)$

$\frac{\partial y}{\partial \underline{\Psi}_{i j}}=\frac{q_{j} g_{j} \frac{\underline{\eta}_{j}}{\underline{\Psi}_{i j}} \sum_{k=1}^{2} \underline{\eta}_{j}-y q_{j} \frac{\underline{\eta}_{j}}{\underline{\Psi}_{i j}}}{\sum_{j=1}^{2} \underline{\Psi}_{1 i} \underline{\Psi}_{2 j}}$

$\frac{\partial y}{\partial \bar{\Psi}_{i j}}=\frac{\left(1-q_{j}\right) g_{j} \frac{\bar{\eta}_{j}}{\bar{\Psi}_{i j}} \sum_{k=1}^{2} \bar{\eta}_{j}-y\left(1-q_{j}\right) \frac{\bar{\eta}_{j}}{\bar{\Psi}_{i j}}}{\sum_{j=1}^{2} \bar{\Psi}_{1 i} \bar{\Psi}_{2 j}}$

Other gradient calculations in the conclusion part of the rules are summarized as follows:

$\frac{\partial E}{\partial w_{j}}=\frac{\partial E}{\partial y} v_{j} \prod_{i=1}^{n} \frac{\sin \left(\frac{x_{i}-b_{i j}}{c_{i j}}\right)}{\left(\frac{x_{i}-b_{i j}}{c_{i j}}\right)}$

$\frac{\partial E}{\partial b_{i j}}=\frac{\partial E}{\partial y} v_{j} w_{j} m \frac{\left(\frac{x_{i}-b_{i j}}{c_{i j}}\right)}{\sin \left(\frac{x_{i}-b_{i j}}{c_{i j}}\right)}$

$m=\prod_{k=1}^{n} \frac{\sin \left(\frac{x_{k}-b_{k j}}{c_{k j}}\right)}{\left(\frac{x_{k}-b_{k j}}{c_{k j}}\right)}\left(\frac{-\cos \left(\left(x_{j}-b_{i j}\right) / c_{i j}\right)}{x_{j}-b_{i j}}+\frac{q \sin \left(\left(x_{j}-b_{i j}\right) / c_{i j}\right)}{\left(x_{j}-b_{i j}\right)^{2}}\right)$

$$
\begin{aligned}
& \frac{\partial E}{\partial c_{i j}}=\frac{\partial E}{\partial y} v_{j} w_{j} u \frac{\left(\frac{x_{i}-b_{i j}}{c_{i j}}\right)}{\sin \left(\frac{x_{i}-b_{i j}}{c_{i j}}\right)} \\
& u=\prod_{k=1}^{n} \frac{\sin \left(\frac{x_{k}-b_{k j}}{c_{k j}}\right)}{\left(\frac{x_{k}-b_{k j}}{c_{k j}}\right)}\left(\frac{-\cos \left(\left(x_{j}-b_{i j}\right) / c_{i j}\right)}{c_{i j}}+\frac{\sin \left(\left(x_{j}-b_{i j}\right) / c_{i j}\right)}{x_{j}-b_{i j}}\right) \\
& \frac{\partial E}{\partial q_{j}}=\frac{\partial E}{\partial y}\left(\frac{\eta_{j}}{\sum_{l=1}^{t}}-\frac{\bar{\eta}_{l}}{\sum_{l=1}^{t} \bar{\eta}_{l}}\right)\left(w_{j} \prod_{i=1}^{n} \frac{\sin \left(\frac{x_{i}-b_{i j}}{c_{i j}}\right)}{\left(\frac{x_{i}-b_{i j}}{c_{i j}}\right)}+p_{j}\right) \\
& \left.\frac{\partial E}{\partial p_{j}}=\frac{\partial E}{\partial y} v_{j}\right) \\
& v_{j}=\frac{q_{j} \underline{\eta_{j}}}{\sum_{i=1}^{t}}+\frac{\left(1-q_{j}\right) \bar{\eta}_{j}}{\sum_{i=1}^{t} \bar{\eta}_{i}}
\end{aligned}
$$

\section{BRIEF DESCRIPTIONS OF OTHER CLASSIFICATION METHODS}

In this paper, the proposed T2FMWNN model is compared other models such as multilayer perceptron (MLP), radial basis function network (RBFN), fuzzy unordered rule induction algorithm (FURIA), Bayes network algorithm, J48 decision tree algorithm. Brief description of these models is explained below:

\section{A. Multilayer Perceptron Network}

Multilayer perceptron network is one of the basic models in artificial neural networks. A typical MLP network has three type of layers: input layer, hidden layer, output layer. The number of hidden layers depend the design. More than one hidden layer is possible. Input layer transmits the inputs to hidden layer. The output layer calculates the MLP output. Different activation functions can be used such as sigmoid or tangent hyperbolic. MLP networks are used in different applications such as time series prediction, classification and control. In this paper, it is used to classification of the breast cancer dataset.

\section{B. Radial Basis Function Network}

RBFN is a type of neural network which uses radial basis functions in its layers. It has total three layers [38]. The first layer is input layer. The second layer is the radial basis function layer, which calculates the distance between radial basis neuron and the input vector. The last layer calculates the output of the RBFN. RBFNs are very good in function learning. Gaussian function can be used as radial basis function in these networks. 


\section{Fuzzy Unordered Rule Induction Algorithm}

FURIA is a fuzzy rule based classification algorithm, which extends the RIPPER algorithm [39]. This algorithm gives fuzzy rules instead of conventional rules. It is more successful than RIPPER algorithm and decision tree algorithm [39]. The conventional rule learners generally learn ordered set of rules however FURIA learns underset set of rules.

\section{Bayesian Network Classification Algorithm}

Bayesian networks are directed acyclic graphs that shows the joint probability between the variables [40]. Vertices represent the variables and edges represent the correlations between variables. Bayesian networks can be trained using minimum description length principle. In [40], it is shown that Bayesian networks give better results than naïve Bayes algorithms in some datasets. However, it can give worse results in other datasets. Thus, the success of the Bayesian networks depends on the dataset.

\section{E. J48 Decision Tree Algorithm}

Decision tree algorithm constructs a tree structure in order to classify the object in the dataset [41]. It calculates entropy of each attribute in the dataset and the attribute with the greatest entropy is brought to top of the tree. Then the entropy is again calculated from the remaining attributes and decision tree construction continues.

\section{BREAST CANCER Classification}

The proposed model is used to classification breast cancer dataset which is obtained from the University of Wisconsin Hospitals, Madison from Dr. William H. Wolberg [42, 43]. This dataset has 9 inputs. This dataset has two classes: sleeping cancer lump (benign) and dangerous cancer lump (malignant). In this paper, first 400 row in dataset is selected as training set and remaining 299 row is selected to test the model.

The proposed T2FMWNN model gives continuous output. In order to use this model in breast cancer classification, the output value below 3 is considered as benign (actual value 2 in dataset) and the output value above 3 is considered as malignant (actual value is 3 in dataset). The dataset has 9 inputs. The percentage of the correctly classified instances is shown in the Table I. The proposed T2FMWNN model is most successful model in breast cancer classification among MLP, RBF, FURIA, Bayes Network, Decision Tree, T1FMWNN and T2FMWNN.

Table I. Percentage of the Correctly Classified Instances According to

\begin{tabular}{|c|c|c|}
\hline Alaorithm & Train & Test \\
\hline MLP & $94.25 \%$ & $97.32 \%$ \\
\hline RBFN & $93.5 \%$ & $96.65 \%$ \\
\hline FURIA & $93.75 \%$ & $96.99 \%$ \\
\hline $\begin{array}{ll}\text { Bayes } & \text { Network } \\
\text { Learning } & \\
\end{array}$ & $94 \%$ & $97.32 \%$ \\
\hline Decision Tree (J48) & $94.25 \%$ & $97.32 \%$ \\
\hline T1FMWNN & $94.75 \%$ & $97.65 \%$ \\
\hline T2FMWNN & $94.5 \%$ & $97.99 \%$ \\
\hline
\end{tabular}

Confusion matrices for MLP network, RBF network, FURIA, Bayes Network, Decision Tree, T1FMWNN and T2FMWNN are shown in Table II, Table III, Table IV, Table V, Table VI, Table VII, Table VIII respectively.
Table II. Confusion Matrix for MLP Network

\begin{tabular}{|l|l|l|}
\hline Real & MLP Output \\
\hline & $\boldsymbol{a}$ & $\boldsymbol{b}$ \\
\hline a=benign & 223 & 6 \\
\hline b=malignant & 2 & 68 \\
\hline
\end{tabular}

Table III. Confusion Matrix for RBF Network

\begin{tabular}{|l|l|l|}
\hline Real & RBF Output \\
\hline & $\boldsymbol{a}$ & $\boldsymbol{b}$ \\
\hline $\boldsymbol{a}=$ =benign & 221 & 8 \\
\hline $\boldsymbol{b}=$ malignant & 2 & 68 \\
\hline
\end{tabular}

Table IV. Confusion Matrix for FURIA

\begin{tabular}{|l|l|l|}
\hline Real & \multicolumn{3}{|l|}{ FURIA Output } \\
\hline & a & b \\
\hline a=benign & 223 & 6 \\
\hline b=malignant & 3 & 67 \\
\hline
\end{tabular}

Table V. Confusion Matrix for Bayes Network

\begin{tabular}{|l|l|l|}
\hline Real & \multicolumn{2}{|l|}{ Bayes Network Output } \\
\hline & $\boldsymbol{a}$ & $\boldsymbol{b}$ \\
\hline a=benign & 223 & 6 \\
\hline $\boldsymbol{b}=$ malignant & 2 & 68 \\
\hline
\end{tabular}

Table VI. Confusion Matrix for Decision Tree

\begin{tabular}{|l|l|l|}
\hline Real & Decision Tree Output \\
\hline & $\boldsymbol{a}$ & $\boldsymbol{b}$ \\
\hline $\boldsymbol{a}=$ benign & 222 & 7 \\
\hline $\boldsymbol{b}=$ malignant & 1 & 69 \\
\hline
\end{tabular}

Table VII. Confusion Matrix for T1FMWNN

\begin{tabular}{|l|l|l|}
\hline Real & \multicolumn{2}{|l|}{ T1FMWNN Output } \\
\hline & $\boldsymbol{a}$ & $\boldsymbol{b}$ \\
\hline a=benign & 225 & 4 \\
\hline $\boldsymbol{b}=$ malignant & 3 & 67 \\
\hline
\end{tabular}

Table VIII. Confusion Matrix for T2FMWNN

\begin{tabular}{|l|l|l|}
\hline Real & T2FMWNN Output \\
\hline & $\boldsymbol{a}$ & $\boldsymbol{b}$ \\
\hline a=benign & 225 & 4 \\
\hline $\boldsymbol{b}=$ malignant & 2 & 68 \\
\hline
\end{tabular}

Sensitivity, specifity and F-1 score is calculated for all algorithms. These measures are calculated from confusion matrices shown in Fig. 3.

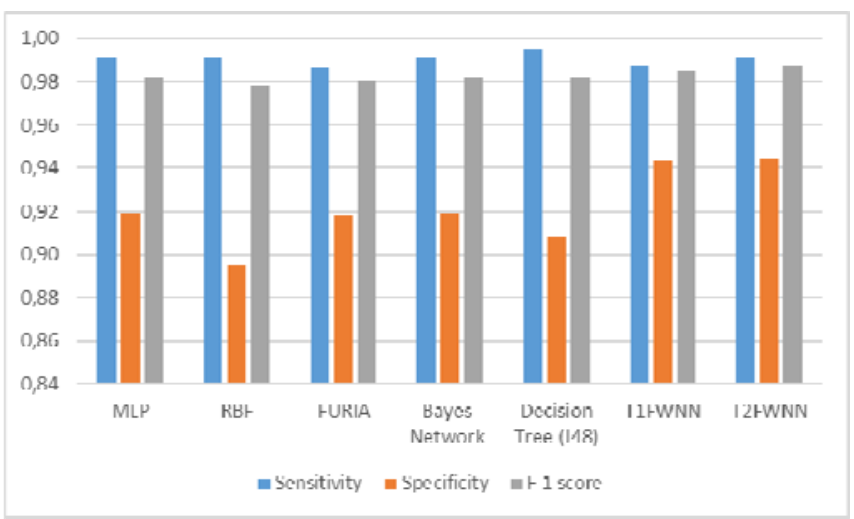

Fig. 3. Sensitivity, specifity and F-1 score for all algorithms 


\section{Conclusion}

In this paper, a new T2FMWNN model is proposed in order to classify the breast cancer data. This new model uses Shannon wavelet functions in its processing units. Type-2 membership functions can catch uncertainty in the data more precisely according the other type-1 membership functions. Therefore, the proposed model gives good results in classification. According to accuracy, specificity and F-1 scores, T2FMWNN model gives the best result among MLP network, RBF network, Bayesian network learning, decision tree and T1FMWNN models. The proposed T2FMWNN model can be applied many other problems such as other classification, function learning, system identification and control problems.

\section{REFERENCES}

[1] T. S. Subashini, V. Ramalingam, and S. Palanivel, "Breast mass classification based on cytological patterns using RBFNN and SVM," Expert Systems with Applications, vol. 36, pp. 5284-5290, Apr 2009.

[2] M. Karabatak and M. C. Ince, "An expert system for detection of breast cancer based on association rules and neural network," Expert Systems with Applications, vol. 36, pp. 3465-3469, Mar 2009.

[3] M. R. Mohebian, H. R. Marateb, M. Mansourian, M. A. Mananas, and F. Mokarian, "A Hybrid Computer-aideddiagnosis System for Prediction of Breast Cancer Recurrence (HPBCR) Using Optimized Ensemble Learning," Computational and Structural Biotechnology Journal, vol. 15, pp. 75-85, 2017.

[4] T. Ince, S. Kiranyaz, J. Pulkkinen, and M. Gabbouj, "Evaluation of global and local training techniques over feed-forward neural network architecture spaces for computer-aided medical diagnosis," Expert Systems with Applications, vol. 37, pp. 8450-8461, Dec 2010.

[5] T. Nguyen, A. Khosravi, D. Creighton, and S. Nahavandi, "Medical data classification using interval type-2 fuzzy logic system and wavelets," Applied Soft Computing, vol. 30, pp. 812-822, May 2015.

[6] T. Nguyen, A. Khosravi, D. Creighton, and S. Nahavandi, "Classification of healthcare data using genetic fuzzy logic system and wavelets," Expert Systems with Applications, vol. 42, pp. 2184-2197, Mar 2015.

[7] A. Ghosh, B. C. Dhara, and R. K. De, "Selection of genes mediating certain cancers, using a neuro-fuzzy approach," Neurocomputing, vol. 133, pp. 122-140, Jun 102014.

[8] N. Leema, H. K. Nehemiah, and A. Kannan, "Neural network classifier optimization using Differential Evolution with Global Information and Back Propagation algorithm for clinical datasets," Applied Soft Computing, vol. 49, pp. 834-844, Dec 2016.

[9] S. Horikawa, T. Furuhashi, and Y. Uchikawa, "On Fuzzy Modeling Using Fuzzy Neural Networks with the Backpropagation Algorithm," IEEE Transactions on Neural Networks, vol. 3, pp. 801-806, Sep 1992.

[10] J. S. R. Jang, "Anfis - Adaptive-Network-Based Fuzzy Inference System," IEEE Transactions on Systems Man and Cybernetics, vol. 23, pp. 665-685, May-Jun 1993.

[11] C. F. Juang and C. T. Lin, "An on-line self-constructing neural fuzzy inference network and its applications," IEEE Transactions on Fuzzy Systems, vol. 6, pp. 12-32, Feb 1998.

[12] J. Kim and N. Kasabov, "HyFIS: adaptive neuro-fuzzy inference systems and their application to nonlinear dynamical systems," Neural Networks, vol. 12, pp. 13011319, Nov 1999.

[13] G. J. Klir and B. Yuan, Fuzzy Sets and Fuzzy Logic Theory and Applications: Prentice Hall, 1995.

[14] O. Castillo and P. Melin, Type-2 Fuzzy Logic: Theory and Applications vol. 223: Springers Berlin Heidelberg, 2008.

[15] Q. L. Liang and J. M. Mendel, "Interval type-2 fuzzy logic systems: Theory and design," IEEE Transactions on Fuzzy Systems, vol. 8, pp. 535-550, Oct 2000.

[16] R. A. Aliev, W. Pedrycz, B. G. Guirimov, R. R. Aliev, U. Ilhan, M. Babagil, et al., "Type-2 fuzzy neural networks with fuzzy clustering and differential evolution optimization," Information Sciences, vol. 181, pp. 15911608, May 12011.

[17] J. R. Castro, O. Castillo, P. Melin, and A. Rodriguez-Diaz, "A hybrid learning algorithm for a class of interval type-2 fuzzy neural networks," Information Sciences, vol. 179, pp. 2175-2193, Jun 132009.

[18] C. F. Juang and Y. W. Tsao, "A Self-Evolving Interval Type-2 Fuzzy Neural Network With Online Structure and Parameter Learning," IEEE Transactions on Fuzzy Systems, vol. 16, pp. 1411-1424, Dec 2008.

[19] G. M. Mendez and M. D. Hernandez, "Hybrid learning mechanism for interval A2-C1 type-2 non-singleton type2 Takagi-Sugeno-Kang fuzzy logic systems," Information Sciences, vol. 220, pp. 149-169, Jan 202013.

[20] S. W. Tung, C. Quek, and C. Guan, "eT2FIS: An Evolving Type-2 Neural Fuzzy Inference System," Information Sciences, vol. 220, pp. 124-148, Jan 202013.

[21] C. H. Wang, C. S. Cheng, and T. T. Lee, "Dynamical optimal training for interval type-2 fuzzy neural network (T2FNN)," IEEE Transactions on Systems Man and Cybernetics Part B-Cybernetics, vol. 34, pp. 1462-1477, Jun 2004.

[22] R. H. Abiyev, O. Kaynak, and E. Kayacan, "A type-2 fuzzy wavelet neural network for system identification and control," Journal of the Franklin Institute-Engineering and Applied Mathematics, vol. 350, pp. 1658-1685, Sep 2013.

[23] F. J. Lin and P. H. Chou, "Adaptive Control of Two-Axis Motion Control System Using Interval Type-2 Fuzzy Neural Network," IEEE Transactions on Industrial Electronics, vol. 56, pp. 178-193, Jan 2009.

[24] J. R. Castro, O. Castillo, P. Melin, O. Mendoza, and A. Rodriguez-Diaz, "An Interval Type-2 Fuzzy Neural Network for Chaotic Time Series Prediction with CrossValidation and Akaike Test," in Soft Computing for Intelligent Control and Mobile Robotics. vol. 318, ed, 2011, pp. 269-285.

[25] M. Singh, S. Srivastava, M. Hanmandlu, and J. R. P. Gupta, "Type-2 fuzzy wavelet networks (T2FWN) for system identification using fuzzy differential and Lyapunov stability algorithm," Applied Soft Computing, vol. 9, pp. 977-989, Jun 2009.

[26] C. Y. Yeh, W. H. R. Jeng, and S. J. Lee, "Data-Based System Modeling Using a Type-2 Fuzzy Neural Network with a Hybrid Learning Algorithm," IEEE Transactions on Neural Networks, vol. 22, pp. 2296-2309, Dec 2011.

[27] C. F. Liu, C. Y. Yeh, and S. J. Lee, "Application of type-2 neuro-fuzzy modeling in stock price prediction," Applied Soft Computing, vol. 12, pp. 1348-1358, Apr 2012.

[28] O. Castillo, R. Martinez-Marroquin, P. Melin, F. Valdez, and J. Soria, "Comparative study of bio-inspired algorithms applied to the optimization of type- 1 and type2 fuzzy controllers for an autonomous mobile robot," Information Sciences, vol. 192, pp. 19-38, Jun 12012.

[29] P. Melin, L. Astudillo, O. Castillo, F. Valdez, and M. Garcia, "Optimal design of type-2 and type-1 fuzzy tracking controllers for autonomous mobile robots under perturbed torques using a new chemical optimization 
paradigm," Expert Systems with Applications, vol. 40, pp. 3185-3195, Jun 152013.

[30] P. Melin and O. Castillo, "A review on the applications of type-2 fuzzy logic in classification and pattern recognition," Expert Systems with Applications, vol. 40, pp. 5413-5423, Oct 12013.

[31] Y. Oysal and S. Yilmaz, "An adaptive wavelet network for function learning," Neural Computing \& Applications, vol. 19, pp. 383-392, Apr 2010.

[32] S. Yilmaz and Y. Oysal, "Fuzzy Wavelet Neural Network Models for Prediction and Identification of Dynamical Systems," IEEE Transactions on Neural Networks, vol. 21, pp. 1599-1609, Oct 2010.

[33] Y. Oysal and S. Yilmaz, "An Adaptive Fuzzy Wavelet Network with Gradient Learning for Nonlinear Function Approximation," Journal of Intelligent Systems, vol. 23, pp. 201-2012, 2014.

[34] S. Yilmaz, "Multilayer Dynamic Fuzzy Neural Network Design for Control and System Identification Applications," PhD PhD Thesis, Computer Engineering, Anadolu University, 2014.

[35] R. H. Abiyev, "A Type-2 Fuzzy Wavelet Neural Network for Time Series Prediction," in IEA/AIE'10 Proceedings of the 23rd international conference on Industrial engineering and other applications of applied intelligent systems, 2010, pp. 518-527.

[36] C. F. Juang, R. B. Huang, and Y. Y. Lin, "A Recurrent Self-Evolving Interval Type-2 Fuzzy Neural Network for
Dynamic System Processing," IEEE Transactions on Fuzzy Systems, vol. 17, pp. 1092-1105, Oct 2009.

[37] J. Nocedal and S. J. Wright, Numerical optimization, 2nd ed. New York: Springer, 2006.

[38] M. J. L. Orr, "Introduction to Radial Basis Function Networks," C. f. C. S. U. o. Edinburgh, Ed., ed. Scotland, 1996.

[39] J. Huhn and E. Hullermeier, "FURIA: an algorithm for unordered fuzzy rule induction," Data Mining and Knowledge Discovery, vol. 19, pp. 293-319, Dec 2009.

[40] N. Friedman, D. Geiger, and M. Goldszmidt, "Bayesian network classifiers," Machine Learning, vol. 29, pp. 131163, Nov-Dec 1997.

[41] M. Kantardzic and MyiLibrary. (2011). Data mining concepts, models, methods, and algorithms (2nd ed.). Available:

https://login.ezproxy1.lib.asu.edu/login?url=http://lib.myil ibrary.com/detail.asp?id=323974

[42] O. L. Mangasarian, W. N. Street, and W. H. Wolberg, "Breast-Cancer Diagnosis and Prognosis Via LinearProgramming," Operations Research, vol. 43, pp. 570577, Jul-Aug 1995.

[43] O. L. Mangasarian and W. H. Wolberg, "Cancer diagnosis via linear programming," SIAM News, vol. 23, pp. 1-18, 1990. 